\title{
Sperm-associated antigen 4 (SPAG4) as a new cancer marker interacts with Nesprin3 to regulate cell migration in lung carcinoma
}

\author{
YING JI $^{1 *}$, JINQUAN JIANG $^{1 *}$, LIHUA HUANG $^{2}$, WEI FENG $^{1}$, \\ ZHANG ZHANG ${ }^{1}$, LONGYU JIN ${ }^{1}$ and XIAOWEI XING ${ }^{2}$ \\ ${ }^{1}$ Department of Cardiothoracic Surgery, ${ }^{2}$ Center for Medical Experiments, \\ Third Xiangya Hospital, Central South University, Changsha, Hunan 410013, P.R. China
}

Received August 14,2017; Accepted April 17, 2018

DOI: $10.3892 /$ or.2018.6473

\begin{abstract}
Lung cancer is the most common cause of cancer-related deaths, and early diagnosis and targeted therapy are extremely important in the treatment of this disease. Sperm-associated antigen 4 (SPAG4) was recently found to be a novel cancer biomarker. In the present study, the expression of SPAG4 was revealed to be high in lung adenocarcinoma tissues as determined by western blotting and immunohistochemistry. SPAG4 knockdown by RNAi efficiently reduced the migration of the lung cancer A549 cells in vitro. Mechanistically, SPAG4 exerted its tumor promoting functions by interacting with Nesprin3 as determined by co-immunoprecipitation (Co-IP) and bimolecular fluorescence complementation (BiFC) techniques. In addition, immunofluorescence revealed that the level of SPAG4 in lung cancer cells could affect the location and expression of Nesprin3. Furthermore, silencing of Nesprin3 reduced the migration of A549 cells and we provided evidence to demonstrate that SPAG4 acted as a positive regulator of Nesprin3. The results revealed that SPAG4, in cooperation with Nesprin3, has a fundamental pathological function in the migration of lung carcinoma cells, and the SPAG4 gene may be useful for the clinical diagnosis and new treatment strategies in patients with lung cancer.
\end{abstract}

Correspondence to: Professor Xiaowei Xing, Center for Medical Experiments, Third Xiangya Hospital, Central South University, 138 Tongzipo Road, Yuelu, Changsha, Hunan 410013, P.R. China E-mail: davy2222@163.com

Professor Longyu Jin, Department of Cardiothoracic Surgery, Third Xiangya Hospital, 138 Tongzipo Road, Yuelu, Changsha, Hunan 410013, P.R. China

E-mail: jinlongyu11@163.com

${ }^{*}$ Contributed equally

Key words: sperm-associated antigen 4, lung cancer, Nesprin3, linker of nucleoskeleton and cytoskeleton complex, mechanism

\section{Introduction}

Lung cancer is the most common malignancy and the leading cause of cancer-related deaths (1). Its occurrence and development is a complex process involving oxygen stress, uncontrolled proliferation, apoptosis, cell transformation and inflammation. With the development of molecular biology, immunotherapy and molecular targeted therapy, significant breakthroughs in the molecular mechanisms of lung cancer have been achieved in recent years. A better understanding of the molecular mechanisms of lung cancer may provide an effective way of improving the prevention, diagnosis, treatment and prognosis of patients with lung cancer.

In clinical diagnosis, pathologists often assess the tumor grade based on the morphology of the nucleus. Components of the nuclear envelope have essential roles in many aspects of cell function and may be involved in tumor development and progression. A series of protein-protein interactions take place on the nuclear envelope which affect tumor cell biology (2). The linker of nucleoskeleton and cytoskeleton (LINC) complex has been suggested to anchor both the nuclear membranes and nuclear lamina to the cytoskeleton (3-5). The KASH domain of the Nesprin family of proteins and the SUN domain of the SUN family of proteins interact in the perinuclear space, and play a major role in the LINC complex (5-8). The SUN family of proteins which are anchored on the inner nuclear membrane connect with the lamina and chromosomes, and the Nesprin family of proteins positioned on the outer nuclear membrane connect with the cytoskeleton proteins $(9,10)$. The LINC complex functions in neurogenesis, gametogenesis and retina formation, and LINC defects may contribute to human diseases, such as muscular dystrophy, ataxia, and cancer $(11,12)$.

Some LINC complex proteins have been reported to be specifically expressed in tumors. The methylation of Nesprin1 gene promoter may be associated with the development of lung cancer $(13,14)$. SUN1 and 2 are expressed at low levels in breast cancer (15). Lv et al demonstrated that SUN2 plays a tumor-suppressor role by suppressing the Warburg effect in lung cancer (16). Sperm-associated antigen 4 (SPAG4), first found in mammalian sperm tails, belongs to the SUN 
family of proteins, interacts specifically with the outer dense fiber 27 (ODF27) protein (17), and plays important roles in spermatogenesis and sperm motility. SPAG4 is localized in the inner nuclear membrane and is a mediating protein between the nucleoskeleton and cytoskeleton. Recently, Kennedy et al reported that SPAG4 may be an independent cancer marker (12). Knaup et al revealed that SPAG4, an HIF1 target, was correlated with the prognosis of renal cell carcinoma (18). From these findings, we speculated that SPAG4 may be associated with the development of lung cancer.

In the present study, we examined clinical samples of lung cancer and found that SPAG4 was highly expressed. To determine the possible mechanism of SPAG4 action in lung cancer, experiments were designed to reveal that SPAG4 interacts with Nesprin3, which influences the migration of lung tumor cells. This SPAG4/Nesprin3 interaction may offer new strategies for the diagnosis and treatment of lung cancer.

\section{Materials and methods}

Collection of tissue samples. This study was approved in December 2014 by the Ethics Committee of the Third Xiangya Hospital, Central South University, Changsha, China, and the collection of lung cancer samples was performed in accordance with ethical standards. The signing of the informed consent form was carried out by the members of the test group and the subjects. When the informed consent was signed, the subject was informed about the details of the clinical trial. None of the patients had undergone preoperative intervention therapy or chemotherapy. The tissues were removed during surgery, and those used for western blotting were immediately stored in liquid nitrogen, and those used for immunohistochemistry were stored in $10 \%$ formalin at room temperature. Paired samples of lung cancerous and paracarcinomatous tissues for immunohistochemistry were obtained from 46 patients with lung cancer who underwent lung lobectomy from December 2014 to June 2015 at the Department of Cardiothoracic Surgery, Third Xiangya Hospital of Central South University, Changsha, China. Sixteen of the 46 paired samples were also used for western blotting. The adjacent tissues were obtained from the edge of the lung lobe $(\geq 5 \mathrm{~cm}$ from the carcinoma). These 46 patients included 29 males and 17 females with a mean age of 57.5 years (range, 32-74 years). No distant metastasis was found in the selected patients before surgery. Detailed clinicopathological data such as tumor size, clinical grade [according to tumor-node-metastasis (TNM) stage], histological type, differentiation degree and lymph node metastasis were obtained and summarized. The tumor stage was defined according to the 8th edition of the TNM classification of the Union for International Cancer Control (UICC).

Construction of plasmids. The eukaryotic vector pEGFP-N1 carrying different fragments of SPAG4 was used, and each of these fragments was amplified by polymerase chain reaction (PCR) using the following primers: forward, 5'-CCAㅡ슬 AGCTTGCCACCAGGATGCGGCGAAGCTCCCG-3' (the HindIII site was included) and reverse, 5'-GGGTCGACA AATGGGGCCCCTGTGCACTG-3' (the SalI site was included) for integral SPAG4; forward, 5'-CCAAGCTTG CCACCAGGATGCGGCGAAGCTCCCG-3' (HindIII) and reverse, 5'-GCGTCGACCGGATGGAACAGACCTCCCT-3' (SalI) for the 1-165 amino acid (aa) fragment of SPAG4; forward, 5'-GCAAGCTTATGCCTCCCCCGCGGGTGTTC AAG-3' (HindIIId) and reverse, 5'-GGGTCGACGCATAGT CGGGCTTCCGCAC-3' (SalI) for the 126-260aa fragment of SPAG4; forward, 5'-GCAAGCTTATGGATTTTGTGCGGA AGCCCGAC-3' (HindIII) and reverse, 5'-GGGTCGACA AATGGGGCCCCTGTGCACTG-3' (SalI) for the 232-438aa fragment of SPAG4.

The eukaryotic vector pCMV-C-flag was used to construct the tagged expression plasmid pCMV-C-flag-SPAG4: forward, 5'-CCAAGCTTGCCACCAGGATGCGGCGAAGCTCCCG-3' (the HindIII site was included) and reverse, 5'-GCE TCGAGATGGGGCCCCTGTGCACTGC-3' (the XhoI site was included). The eukaryotic vector pCMV-N-HA was used to construct pCMV-N-HA-Nesprin3: forward, 5'-CATAAG CTTATGAACTCAGCAGCCCCAGGAC-3' (the HindIII site was included) and reverse, 5'-GGGATCCATCCTCCA AAAATCGGAT-3' (the $K p n I$ site was included). The products were sequenced to confirm the identity. The procedure and conditions for PCR were: $95^{\circ} \mathrm{C}$ for $2 \mathrm{~min}$ and $30 \mathrm{sec}$, followed by 35 cycles at $94^{\circ} \mathrm{C}$ for $30 \mathrm{sec}, 62^{\circ} \mathrm{C}$ for $30 \mathrm{sec}$, and $72^{\circ} \mathrm{C}$ for $1 \mathrm{~min}$ and $20 \mathrm{sec}$, and a final extension at $72^{\circ} \mathrm{C}$ for $10 \mathrm{~min}$. All finished vectors were sent to Sangon Biotech Co., Ltd. (Shanghai, China) for gene sequencing.

Cell culture and electric transfection methods. The lung cancer cell line A549 was purchased from the Xiangya Cell Bank, Central South University, Changsha, China. RPMI-1640 medium (Gibco; Thermo Fisher Scientific, Inc., Waltham, MA, USA) with $10 \%$ fetal bovine serum (FBS) was used for cell culture. For electric transfection, the cells were diluted with electric transfection buffer (Eppendorf, Hamburg, Germany) at $0.5-1.5 \times 10^{6}$ cells $/ \mathrm{ml}$, mixed with plasmids $20-30 \mu \mathrm{g} / \mathrm{ml}$, and then placed in electric transfection dishes. The cell dishes were installed on a Multiporator (Eppendorf Electroporator 2510), with the parameters set at $600 \mathrm{~V}$ and $100 \mu \mathrm{sec}$. The transferred cells were cultured in normal medium at $37^{\circ} \mathrm{C}$ in a $5 \% \mathrm{CO}_{2}$ and $95 \%$ humidified air atmosphere. After $20 \mathrm{~h}$, the cells were observed under a fluorescence microscope and images were obtained.

Bimolecular fluorescence complementation (BiFC) assay. To construct recombinant BiFC plasmids, the Nesprin3 and SPAG4 cDNA sequences were respectively amplified (from A549 total mRNA) by PCR using the following primers: forward, 5'-CCAAGCTTGCCACCAGGATGCGGCGAAGC TCCCG-3' (the HindIII site was included) and reverse, 5'-CCE GTACCATGGGGCCCCTGTGCACTGCCCTC-3' (KpnI) for SPAG4; forward, 5'-CATAAGCTTATGAACTCAGCAGCC CCAGGAC-3' (HindIII) and reverse, 5'-GGGATCCATCCT CCAAAAATCGGAT-3' (KpnI) for Nesprin3. Fragments of YFP DNA sequence from the PA7-YFP vector were truncated at residue 155, and were thus designated as YN and YC DNA fragments, using the following primers: forward, 5-'CGG GGTACCCGCTCCATCGCCACGATGGTGAGCAAGGGC GAG-3' (KpnI was included) and reverse, 5'-CCG $\underline{\text { GAA }}$ TTCTTAGGCCATGATATAGACGT TGT-3' (EcoRI) for YN; forward, 5'-CGGGGTACCCGCCCGGCCTGCAAGATCCC GAACGACCTGAAACAGAAGGTCATGAACCACGACAA 
GCAGAAGAACGGCAT-3' (KpnI) and reverse, 5'-CCGGAA TTCTTACTTGTACAGCTCGTCCATGC-3' (EcoRI) for YC. The YN DNA fragment was connected to the 3' (C-terminus) of Nesprin 3 cDNA, and the YC fragment was connected to the 3' (C-terminus) of SPAG4 cDNA. The connected fragments were inserted into the pcDNA3.1(+) vector to generate pcDNA3.1(+)-Nesprin3-YN and pcDNA3.1(+)-SPAG4-YC, respectively. All constructs were verified by sequencing (Sangon Biotech Co., Ltd.).

For the BiFC assay, the cells were suspended in $300 \mu \mathrm{l}$ transfer buffer (Eppendorf) and co-transfected with a mixture of pcDNA3.1(+)-Nesprin3-YN and pcDNA3.1(+)-SPAG4-YC using the electroporation method. Cells were maintained in a $5 \% \mathrm{CO}_{2}$ and $95 \%$ humidified air atmosphere at $37^{\circ} \mathrm{C}$ for $20 \mathrm{~h}$. Finally, the cells were analyzed using a fluorescence microscope (Nikon Corp., Melville, NY, USA).

Establishment of the SPAG4 and Nesprin 3 silenced A549 cell model. The GV248 lentiviral vector (containing neomycin resistant and EGFP genes; Shanghai GeneChem , Co., Ltd., Shanghai, China) carrying three types of SPAG4-shRNA or three types of Nesprin3-shRNA was used to screen for maximum silencing efficiency. We electrically transfected the SPAG4-shRNA or Nesprin3-shRNA vectors into A549 cells to construct the SPAG4/Nesprin3-silenced A549 cell model, and the analogue vector was used as the control. Twenty hours after electric transfection, the A549 cells were cultured in medium with neomycin $(1 \mathrm{mg} / \mathrm{ml})$ for 9 days (5-7 days after all cells in the untransfected group died). Each cell line was then subjected to extensive culture. SPAG4 or Nesprin 3 expression was determined for each group using western blotting.

Western blotting. Tissues or cells were lysed in radioimmunoprecipitation assay (RIPA; Beyotime Institute of Biotechnology, Haimen, China) buffer with protease inhibitors (cOmplete Mini; Roche Diagnostics, Basel, Switzerland) and phenylmethanesulfonyl fluoride (PMSF) (KGP610; Nanjing KeyGen Biotech Co., Ltd., Nanjing, China). After electrophoresis, the proteins were transferred to polyvinylidene fluoride (PVDF) membranes (IPVH10010; EMD Millipore, Billerica, MA, USA). The Bio-Rad electrophoresis system and Wet blotting system (both from Bio-Rad Laboratories, Inc., Hercules, CA, USA) were used to perform the aforementioned steps. Non-specific binding was blocked by immersing the membrane in 5\% non-fat milk for $2 \mathrm{~h}$ at room temperature with shaking. Primary antibodies were applied overnight at $4^{\circ} \mathrm{C}$. The rabbit anti-SPAG4 antibody (cat. no. sc-135414; Santa Cruz Biotechnology, Inc., Dallas, TX, USA) was used at a 1:500 dilution, the rabbit anti-Nesprin3 antibody (cat. no. ab74261; Abcam, Cambridge, UK) was used at a 1:1,000 dilution, the mouse anti-Flag antibody (cat. no. AF519; Beyotime Institute of Biotechnology), the mouse HA-Tag antibody (cat. no. 66006-1-1g) and mouse anti-GAPDH antibody (cat. no. 60001-1-1g) (both from ProteinTech Group, Inc.: Wuhan Sanying Biotechnology, Wuhan, China) were used at a 1:2,000 dilution. Horseradish peroxidase-conjugated anti-mouse (cat. no. 7076s) or anti-rabbit secondary antibodies (cat. no. 7074s) (both from Cell Signaling Technology, Inc., Danvers, MA, USA) were applied at 1:3,000 for $1 \mathrm{~h}$ at $37^{\circ} \mathrm{C}$. Signals were visualized using an enhanced chemiluminescent (ECL) system. Protein band intensity was measured and normalized to GAPDH by ImageJ software.

Immunohistochemical staining. Immunohistochemical staining for the SPAG4 protein was carried out using an SP ready-to-use kit (Beijing Zhongshan Golden Bridge Biotechnology Co., Ltd.: OriGene Technologies, Beijing, China) according to the manufacturer's instructions. The rabbit anti-hSPAG4 antibody was used as the primary antibody according to the manufacturer's instructions. The SPAG4 antibody was diluted at 1:50. Sections were then incubated with biotin-labeled goat anti-rabbit/mouse IgG (Beijing Zhongshan Golden Bridge Biotechnology Co., Ltd.: OriGene Technologies) for $10 \mathrm{~min}$ and subsequently with streptavidin peroxidase for $10 \mathrm{~min}$. The slides were visualized using diaminobenzidine and counterstained with hematoxylin before microscopy. Addition of the primary rabbit anti-SPAG4 antibody was omitted from the protocols for the negative controls.

Three random $\times 200$ microscopic fields containing tumor cells were evaluated by two independent experienced investigators without prior knowledge of the patient disease data; $\geq 100$ tumor cells were examined per field. Two scoring systems including staining intensity and percentage of immunoreactive cells were used. The staining intensity was scored on a semi-quantitative 4-point scale: 0 , negative control; 1 , weak cytoplasmic and nuclear staining (slightly darker than the negative control); 2 , moderate staining; and 3, intense staining (equivalent to or darker than the positive control). The percentage of stained cells was also scored on a semiquantitative 4-point scale: 0 for $<10 \%, 1$ for $10-25 \%, 2$ for $25-50 \%$, and 3 for $>50 \%$. Finally, the scores of staining intensity and the percentage of stained cells were combined and evaluated as follows: $0-1$ as -, 2 as +, 3-4 as ++ and 5-6 as +++.

Co-immunoprecipitation (Co-IP). Protein $(200 \mu \mathrm{g})$ extracted from the transfected cells, $30 \mu 1$ sepharose beads (Santa Cruz Biotechnology, Inc.) and cell lysates [treated with $1 \mathrm{mM}$ DL-dithiothreitol (DTT), $1 \mathrm{mM}$ PMSF, $1 \mu \mathrm{g} / \mathrm{ml}$ aprotinin, $1 \mu \mathrm{g} / \mathrm{ml}$ leupeptin protease inhibitor, $0.5 \%$ NP40] were mixed to a volume of $400 \mu \mathrm{l}$. The cell lysates obtained from the cells were precleared by incubation for $2 \mathrm{~h}$ at $4^{\circ} \mathrm{C}$ on a shaker. The clear supernatant was incubated overnight with control IgG, anti-HA (cat. no. 66006-1-1g; ProteinTech Group, Inc.: Wuhan Sanying Biotechnology) or anti-FLAG antibody (AF519; Beyotime Institute of Biotechnology). The samples were then washed with $0.5 \%$ NP40 cell lysate three times. Immobilized protein complexes were eluted by denaturation in $2 \mathrm{X}$ SDS sample buffer at $95^{\circ} \mathrm{C}$ for $10 \mathrm{~min}$ for subsequent western blotting.

Immunofluorescence. For immunofluorescence, the cells were washed with phosphate-buffered saline (PBS) solution $\left(4^{\circ} \mathrm{C}\right)$ and immediately fixed in cold methanol for $15 \mathrm{~min}$ and permeabilized for $10 \mathrm{~min}$ in $0.2 \%$ Triton X-100 (Beyotime Institute of Biotechnology). Staining antibodies included mouse anti-Nesprin3 at a 1:200 dilution and rabbit anti-SPAG4 at a 1:100 dilution. After washing, the sections were incubated with Cy3-labeled goat anti-rabbit IgG (cat. no. KGAB019; Nanjing KeyGen Biotech Co., Ltd.). Nuclei were counterstained using 

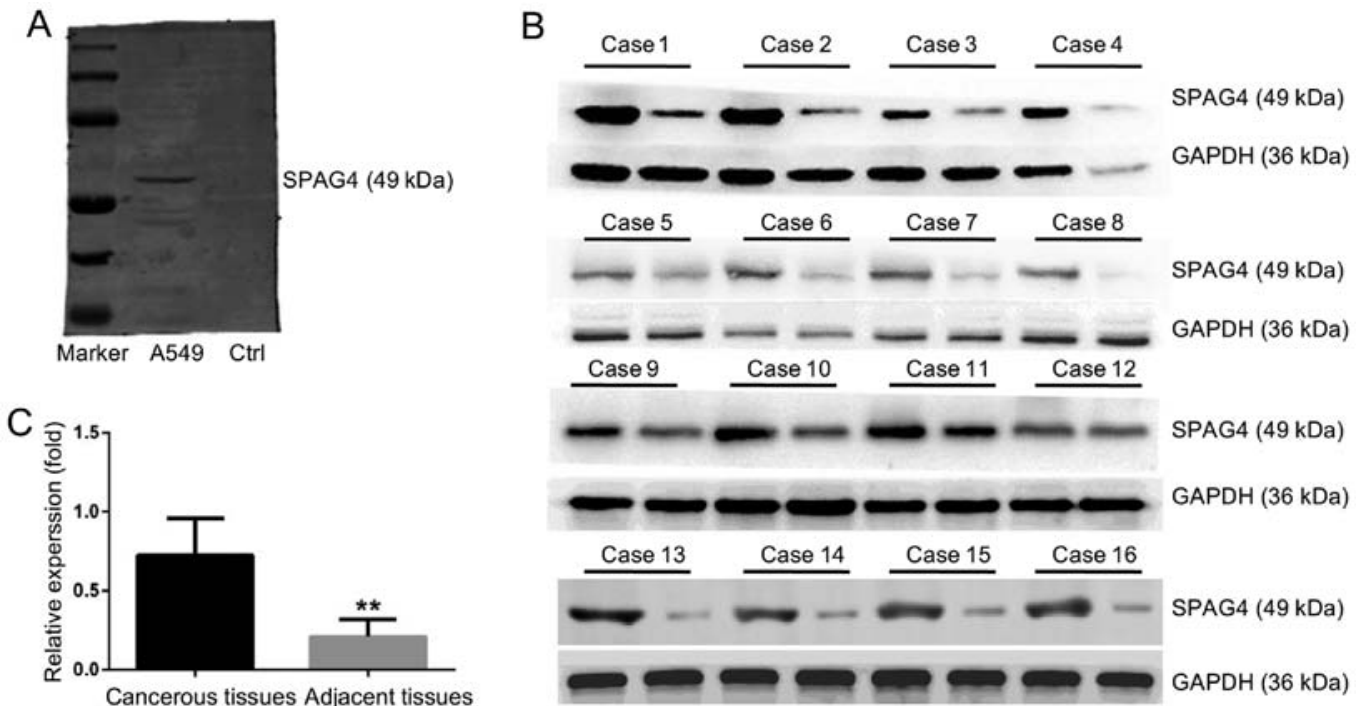

Figure 1. Western blotting of SPAG4 expression in lung carcinoma. (A) Validation of anti-SPAG4 antibody specificity. The lysates of A549 cells (lane 2) and lysis buffer with no protein (blank control, lane 3) were subjected to western blotting with an anti-SPAG4 antibody. (B) Western blotting revealed that SPAG4 was highly expressed in 16 pairs of lung carcinoma tissues. In each case, the left is from lung carcinoma tissue, the right is from adjacent tissue. GAPDH was used as an internal control. (C) The statistical results based on SPAG4 relative expression. Box plots correspond to the mean $\pm \mathrm{SD}$, and the P-value was calculated using the Student's t-test. ${ }^{* *} \mathrm{P}<0.01$. SPAG4, sperm-associated antigen 4.

DAPI (Invitrogen: Thermo Fisher Scientific, Inc., Carlsbad, CA, USA). After staining, the cells were imaged on a Nikon TE300 Eclipse inverted microscope (Nikon Corp.).

Statistical analysis. Statistical analyses were carried out using SPSS version 24.0. Analysis of the association between the expression of SPAG4 and clinicopathological features was performed using Pearson's Chi-square test or Fisher's exact test. All values from the in vitro assays were expressed as the mean \pm SEM or SD of at least three independent experiments or replicates. $\mathrm{P}<0.05$ was considered statistically significant in all tests.

\section{Results}

Increased expression of SPAG4 in lung cancer tissue. Fig. 1A shows the specificity of the anti-SPAG4 antibody. No non-specific bands were detected and the antibody was qualified for immunohistochemistry studies. Fig. 1B and C reveals the high SPAG4 expression in lung carcinoma tissues. Based on the assessment by pathologists, SPAG4 expression as revealed by immunohistochemistry was divided into four levels. The cancer and paracancerous tissue sampling areas are shown in Fig. 2A and B, respectively. The staining of the SPAG4 protein was predominantly localized in the cytoplasm of lung cancer cells. Precise localization of SPAG4 is shown in the immunofluorescence results. The positive rate of SPAG4 expression in cancerous tissues was $69.6 \%$ (32/46), and only $21.7 \%(10 / 46)$ in the corresponding adjacent non-cancerous tissues (Table I). The ratio of SPAG4 positivity was significantly different between the cancerous and adjacent tissues $(\mathrm{P}<0.001)$.

Notably, according to these data, SPAG4 expression was not significantly correlated with age, histological type or tumor size, but was strongly correlated with tumor differentiation grade $(\mathrm{P}=0.014)$, lymph node metastasis $(\mathrm{P}=0.035)$ and
Table I. Differential expression of SPAG4 in cancerous and corresponding adjacent tissues.

\begin{tabular}{lcccc}
\hline \multirow{2}{*}{ Tissues } & \multicolumn{2}{c}{ SPAG4 expression } & \\
\cline { 3 - 4 } & $\begin{array}{c}\text { No. of } \\
\text { cases }\end{array}$ & $\begin{array}{c}\text { Negative } \\
(-/+)\end{array}$ & $\begin{array}{c}\text { Positive } \\
(++/+++)\end{array}$ & $\begin{array}{c}\text { Positive } \\
\text { rate (\%) }\end{array}$ \\
\hline Cancerous & 46 & 14 & 32 & $69.6^{\mathrm{a}}$ \\
Adjacent & 46 & 36 & 10 & 21.7 \\
\hline
\end{tabular}

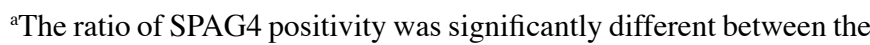
cancerous and adjacent tissues $(\mathrm{P}<0.001)$. SPAG4, sperm-associated antigen 4 .

clinical stage ( $\mathrm{P}=0.008)$ (Table II). These results indicated that SPAG4 was upregulated in lung cancer and its high expression was closely related to tumor cell migration in lung cancer.

SPAG4 promotes the migration of lung cancer cells. Given that SPAG4 expression was upregulated in lung cancer tissues, we then determined whether SPAG4 was involved in regulating the biological behavior of lung cancer cells. Marked downregulation of SPAG4 expression was observed in SPAG4-RNAi-treated A549 cells (Fig. 3A). Immunofluorescence further demonstrated an evident reduction in SPAG4 expression in SPAG4-RNAi-treated A549 cells, demonstrating that the expression of SPAG4 was successfully reduced by the RNAi method (Fig. 3B).

As shown in Table II, SPAG4 may be closely associated with tumor cell migration. The scratch wound assay revealed that cell migration of SPAG4-silenced cells was slower than that of the control (Fig. 3C and D). These results indicated that SPAG4 plays an important role in the migration of lung cancer 

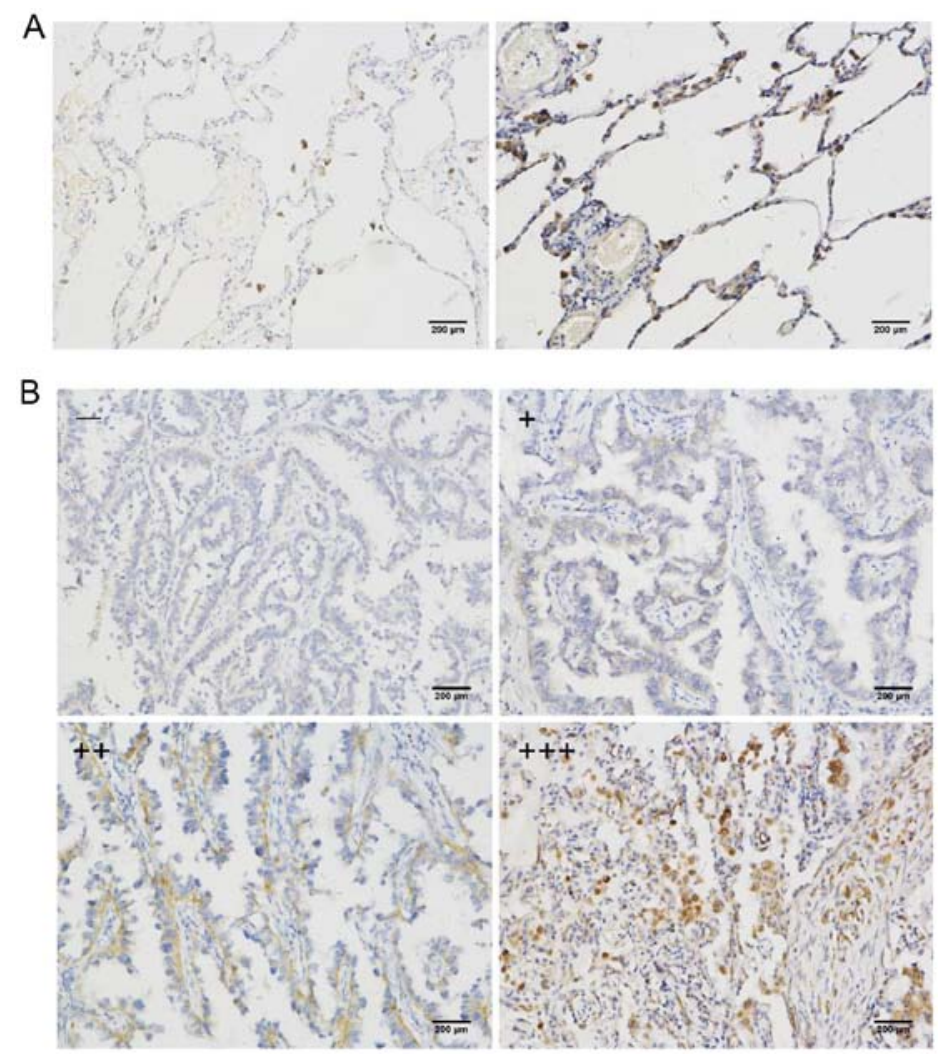

Figure 2. IHC staining of SPAG4 in lung carcinoma tissues. (A) Representative IHC staining of SPAG4 in non-cancerous lung tissues. The sample on the left is negative, and the sample on the right is positive. (B) Representative IHC staining of SPAG4 in lung carcinoma tissues. IHC results were divided into four categories. The basis and method of classification can be found in Materials and methods. SPAG4, sperm-associated antigen 4; IHC, immunohistochemical.
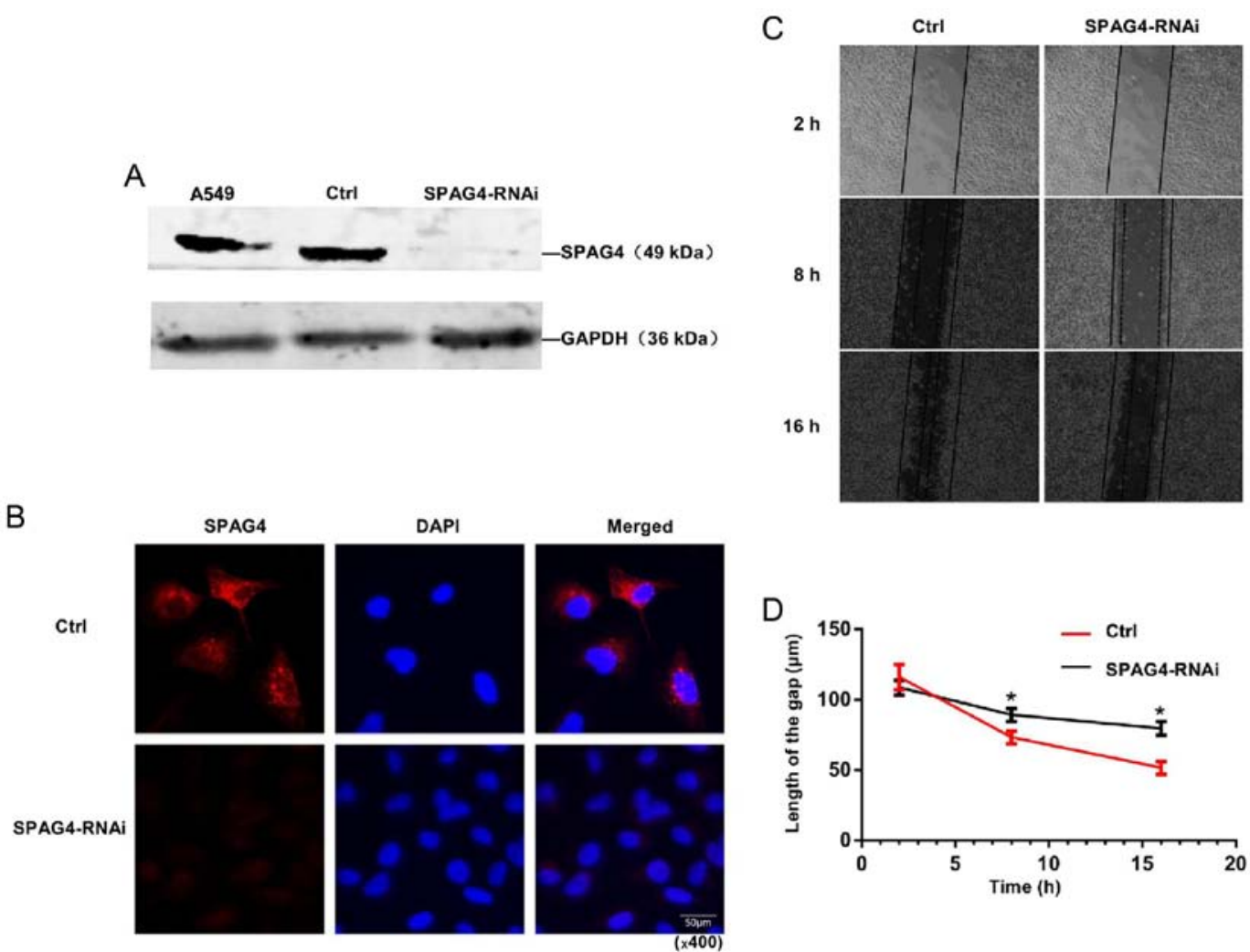

Figure 3. Effects of SPAG4 gene silencing in A549 cells. (A) Western blotting revealed that the SPAG4 gene was successfully silenced by SPAG4-RNAi. (B) Using Cy3 red fluorescence-labeled SPAG4 antibody, SPAG4-silenced cells did not exhibit red fluorescence. (C and D) Scratch wound assay. The images in each group were obtained by microscopy 2,8 and $16 \mathrm{~h}$ after the cells were scratched (magnification, $\mathrm{x} 40$ ). The extension of cells in the SPAG4-RNAi group was slower than those in the control. The line chart corresponds to the mean \pm SEM, and the P-value was calculated using the Student's t-test. ${ }^{*} \mathrm{P}<0.05$. SPAG4, sperm-associated antigen 4. 
Table II. Association between SPAG4 expression and clinicopathological features.

\begin{tabular}{|c|c|c|c|c|c|c|}
\hline \multirow{3}{*}{$\begin{array}{l}\text { Clinicopathological } \\
\text { data }\end{array}$} & \multirow[b]{3}{*}{ Patients } & \multicolumn{4}{|c|}{ SPAG4 expression } & \multirow[b]{3}{*}{ P-value } \\
\hline & & \multicolumn{2}{|c|}{ Negative } & \multicolumn{2}{|c|}{ Positive } & \\
\hline & & - & + & ++ & +++ & \\
\hline Total & 46 & 5 & 9 & 18 & 14 & \\
\hline Age (years) & & & & & & 0.403 \\
\hline$<60$ & 22 & 4 & 4 & 8 & 6 & \\
\hline$>60$ & 24 & 1 & 5 & 10 & 8 & \\
\hline Histological type & & & & & & 0.766 \\
\hline Squamous carcinoma & 15 & 1 & 4 & 5 & 5 & \\
\hline Adenocarcinoma & 31 & 4 & 5 & 13 & 9 & \\
\hline Tissue size $(\mathrm{cm})$ & & & & & & 0.447 \\
\hline $\mathrm{D}<3$ & 16 & 2 & 4 & 6 & 4 & \\
\hline $\mathrm{D}>3$ & 30 & 3 & 5 & 12 & 10 & \\
\hline Clinical grade & & & & & & $0.008^{\mathrm{a}}$ \\
\hline I & 17 & 3 & 6 & 6 & 2 & \\
\hline II & 16 & 2 & 3 & 5 & 6 & \\
\hline III & 13 & 0 & 0 & 7 & 6 & \\
\hline Differentiation degree & & & & & & $0.014^{\mathrm{a}}$ \\
\hline Undifferentiated & 8 & 0 & 0 & 3 & 5 & \\
\hline Poorly differentiated & 13 & 0 & 2 & 5 & 6 & \\
\hline $\begin{array}{l}\text { Moderately/ } \\
\text { well-differentiated }\end{array}$ & 25 & 5 & 7 & 10 & 3 & \\
\hline $\begin{array}{l}\text { Lymph node } \\
\text { metastasis }\end{array}$ & & & & & & $0.035^{\mathrm{a}}$ \\
\hline Yes & 30 & 2 & 4 & 13 & 11 & \\
\hline No & 16 & 3 & 5 & 5 & 3 & \\
\hline
\end{tabular}

${ }^{\mathrm{a}} \mathrm{P}<0.05$, significantly different. SPAG4, sperm-associated antigen 4.

cells, and a higher level of SPAG4 expression is highly related to stronger migration of cancer cells.

Transmembrane helix plays a major role in SPAG4 positioning. To determine the location of SPAG4 protein in lung cancer cells, we generated the pEGFP-N1-SPAG4 plasmid encoding the SPAG4-GFP fusion protein, and then a series of GFP-SPAG4 deletion mutants were generated to identify the region that is critical for localization of SPAG4 to the nuclear envelope or cytoplasm. According to the gene information in NCBI (Fig. 4A), we constructed the full-length and three fragments of SPAG4 into the pEGFP-N1 plasmid, respectively. Each of the four plasmids was transfected into A549 cells, and confocal laser scanning microscopy was used to obtain images (Fig. 4B). The green fluorescence from the full-length SPAG4-transfected cells was located evenly around the nucleus and on one side of the nucleus in the cytoplasm, while the fragment 1-165aa was located in the cytoplasm and not on the nuclear membrane. The fragment 126-260aa, containing the transmembrane helices and the coiled-coil region, was positioned in the membrane structure of the cells. However, its agglomeration was not only on one side of the nucleus but also occurred elsewhere in the cytoplasm. The

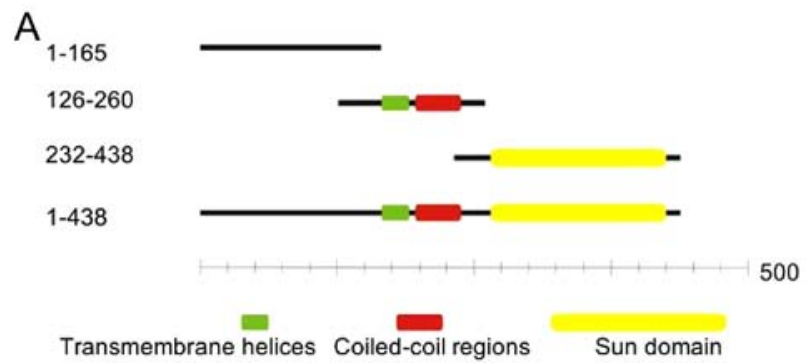

B

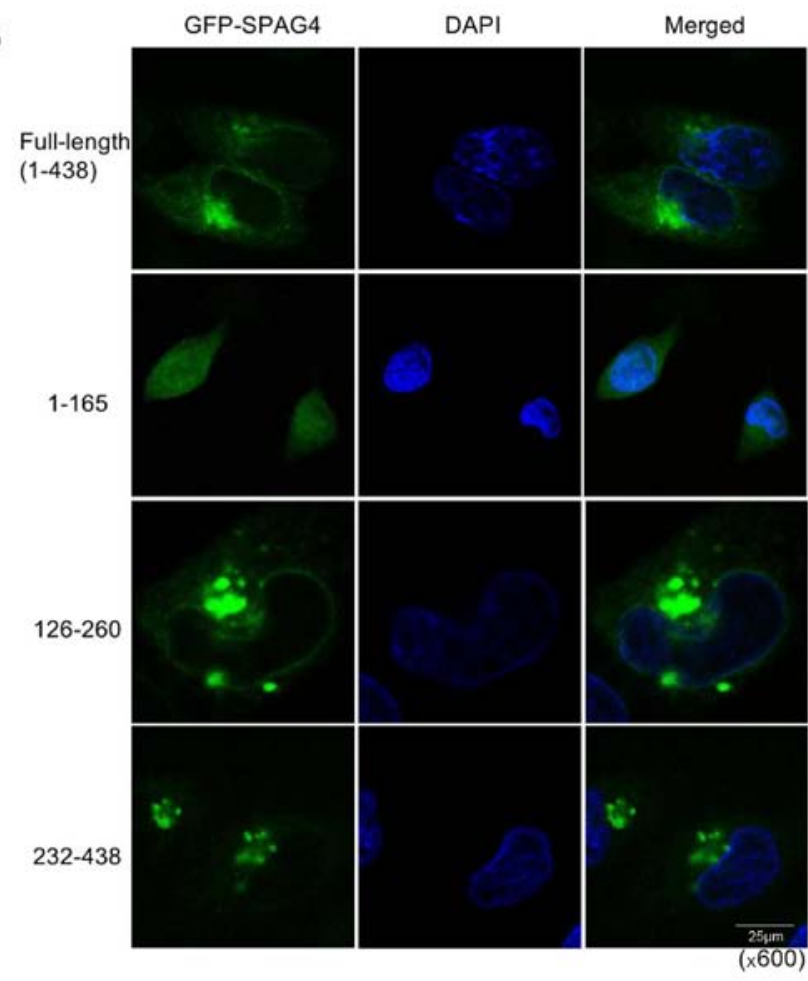

Figure 4. Subcellular localization of SPAG4 and its fragments in A549 cells. (A) Schematic diagram of the three domains of the SPAG4 protein. Different recombinational fragments of SPAG4 were designed to study its function. (B) Plasmids of different SPAG4 fragments were electrically transfected into A549 cells, and the fluorescence distribution was observed under a confocal laser scanning microscope (magnification, x600). SPAG4, sperm-associated antigen 4 .

fragment 232-438aa, containing only the SUN domain, was not only located in the membrane structure, but also on one side of the nucleus in the cytoplasm. It is evident that the C-terminus (SUN domain) of SPAG4 most probably guides it to the nuclear envelope, while the transmembrane helices help it anchor on the inner nuclear envelope.

Identification of Nesprin 3 as an interacting protein of SPAG4. Since it has long been known that Nesprin3 silencing greatly attenuates the migratory flow response with cells moving significantly more slowly in the direction of flow (19), we suspected that SPAG4 may be closely related to Nesprin3, which is located on the outer nuclear membrane and is also a component of the LINC complex.

The Co-IP assay was used to successfully confirm the interaction between SPAG4 and Nesprin3 (Fig. 5A). Following co-transfection with the pCMV-C-flag-SPAG4 and 
A
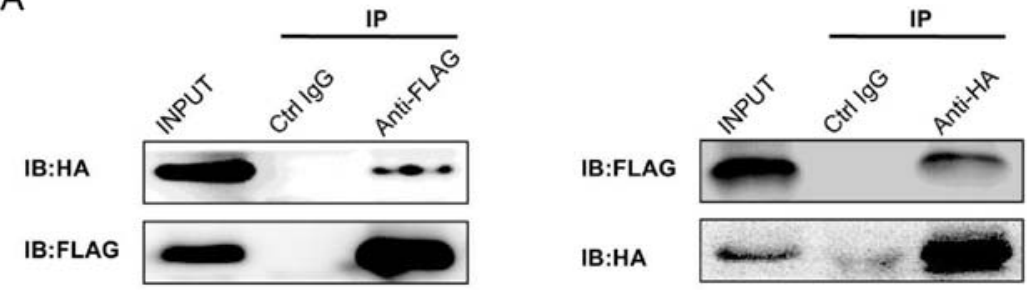

B
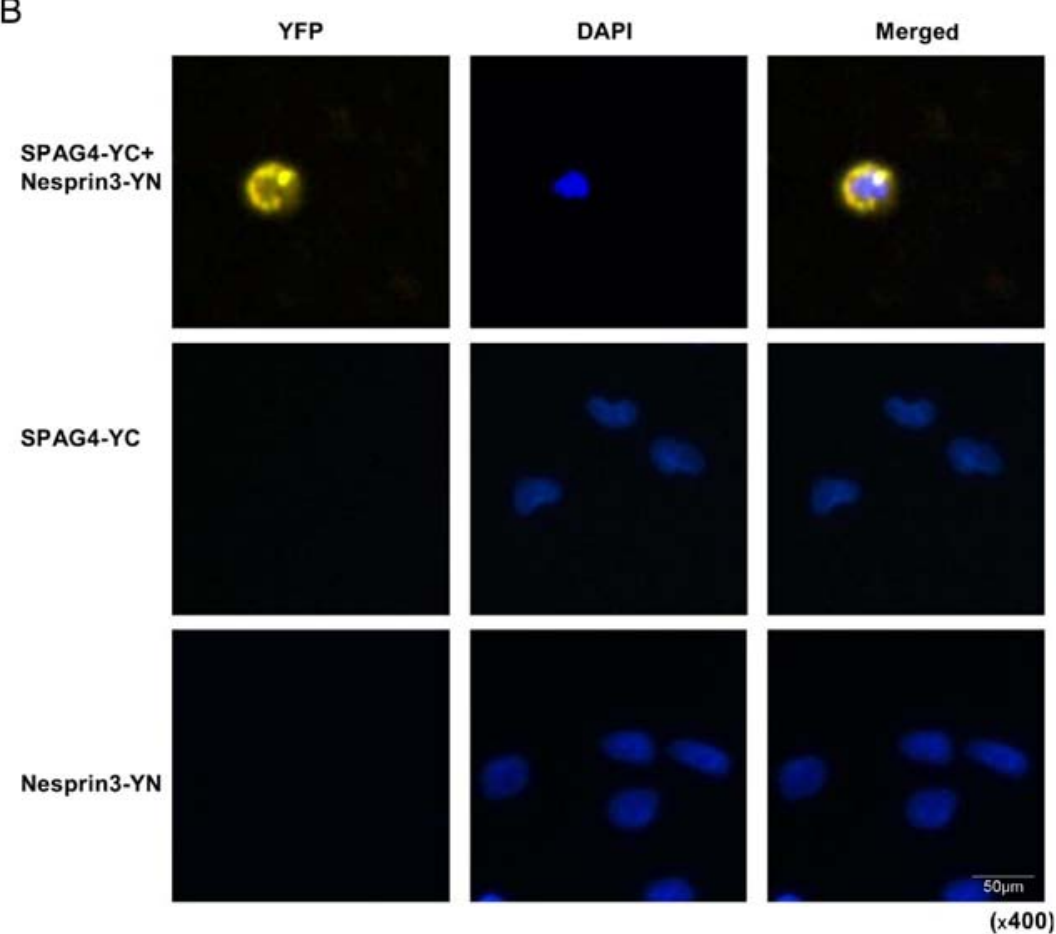

Figure 5. SPAG4 interacts with Nesprin3 in the nuclear envelope. (A) Co-IP of FLAG-SPAG4 with HA-Nesprin3. The lysates of A549 cells co-transfected with the FLAG-SPAG4 and HA-Nesprin3 vectors were subjected to IP as observed in the right panel with an anti-FLAG antibody (lane 3, left), an anti-HA antibody (lane 3, right) or a non-specific control IgG (lane 2) and IB with an anti-HA or anti-FLAG antibody. An input protein (5\%) was used as the control in the IB of the transfected cell lysates (lane 1). (B) The BiFC assay. Cells transfected with SPAG4-YC or Nesprin3-YN alone which acted as control groups exhibited no fluorescence. When SPAG4-YC and Nesprin3-YN plasmids were co-transfected into cells, the YC and YN fragments at the ends were complementary and exhibited yellow fluorescence. It is evident that SPAG4 and Nesprin3 interacted with each other around the nuclear envelope. SPAG4, sperm-associated antigen 4; Co-IP, co-immunoprecipitation; IB, immunoblotted; BiFC, bimolecular fluorescence complementation.

pCMV-N-HA-Nesprin3 vectors, the cell lysates were subjected to the Co-IP assay. In addition, we consolidated this conclusion using the BiFC assay (20). Cells were co-transfected with pcDNA3.1-SPAG4-YC and pcDNA3.1-Nesprin3-YN, and the yellow image around the nuclear envelope indicated the interaction between SPAG4 and Nesprin3 (Fig. 5B).

SPAG4 exerts migration-promoting functions by regulating Nesprin3 in lung cancer. The interaction between SPAG4 and Nesprin 3 was further confirmed by the distribution of Nesprin3 (Fig. 6A). Immunofluorescence staining revealed that Nesprin 3 exhibited strong nuclear membrane localization in A549 cells. When SPAG4 was silenced, Nesprin3 was not completely distributed around the nuclear membrane, and its expression appeared to decrease. As shown in Fig. 6B and C, SPAG4 silencing significantly reduced the expression of Nesprin3.

It appears that SPAG4 affects the migration of lung cancer cells by regulating Nesprin3. Thus, we studied the effect of
Nesprin 3 on the migration of lung cancer cells. We performed the scratch wound assay, and found that Nesprin3-silenced A549 cells (verified by western blotting as shown in Fig. 7A) had slower migration, and exhibited the same trend as SPAG4 (Fig. 7B and C). These results indicated that SPAG4, acting as a positive regulator of Nesprin3, promoted cell migration in lung carcinoma.

\section{Discussion}

SPAG4 is a new potential tumor biomarker, and was found to be significantly increased in lung carcinoma tissues. The present study indicated that downregulation of SPAG4 expression suppressed cell migration. In addition, we validated the interaction between SPAG4 and Nesprin3, and the role of Nesprin3 in migration conformed with the possible link between the two proteins. We found that SPAG4 may regulate the localization and expression of Nesprin 3 at the protein level and further promote the development of lung cancer. 
A
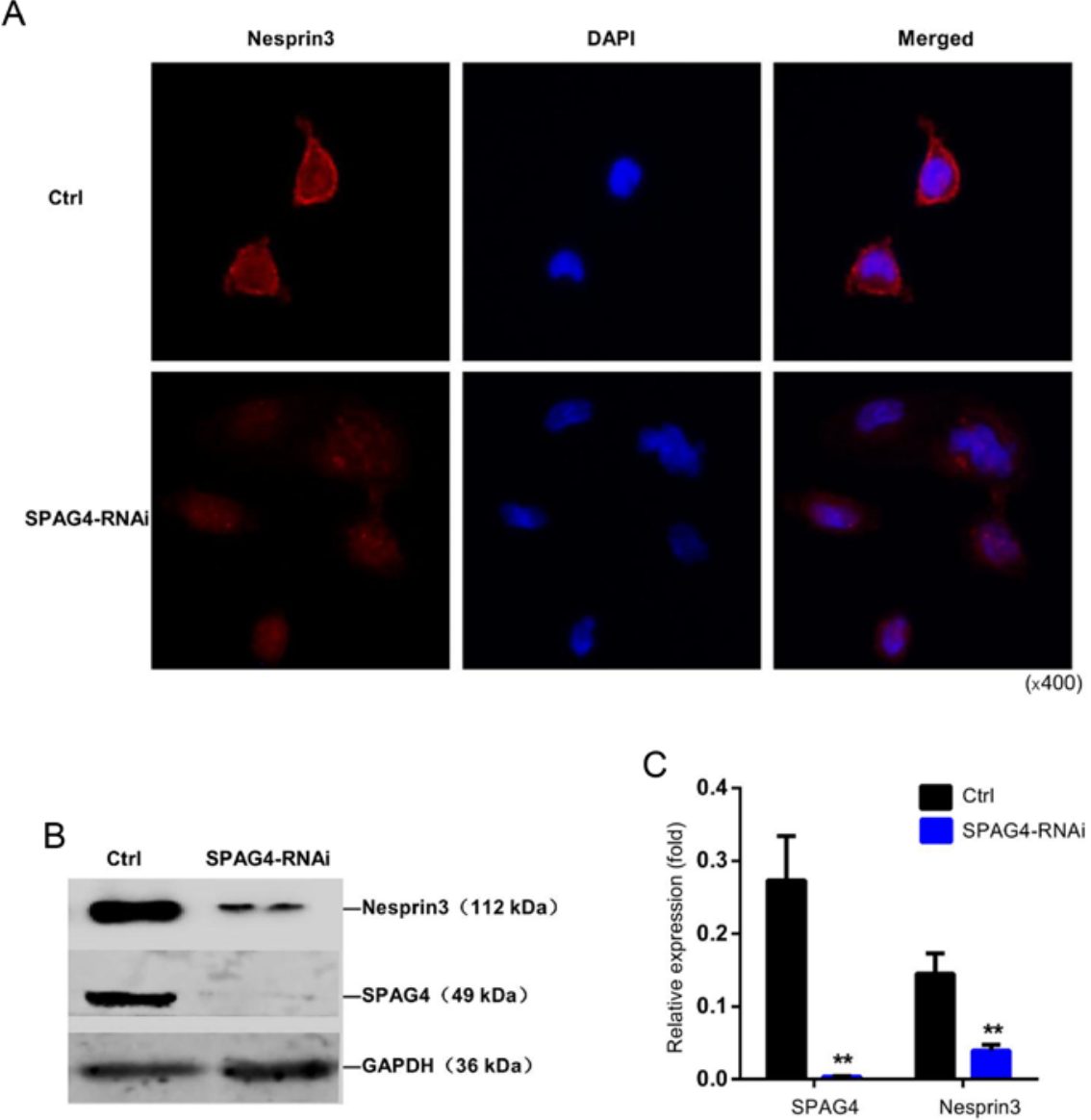

Figure 6. SPAG4 silencing inhibits the distribution and expression of Nesprin3 in lung cancer cells. (A) Nesprin3 localization in SPAG4-RNAi-treated A549 cells and control A549 cells, is displayed using Cy3 red fluorescence-labeled Nesprin3 antibody. (B and C) Protein levels of Nesprin3 and SPAG4 were determined in SPAG4-RNAi-treated A549 cells and control cells using western blotting, respectively. GAPDH was used as an internal control. The bars correspond to the mean \pm SEM. The P-value was calculated using the Student's t-test. ${ }^{* *} \mathrm{P}<0.01$. SPAG4, sperm-associated antigen 4.

This SPAG4/Nesprin3 axis may be useful for the development of new treatment strategies for patients with lung cancer. In the present study, we focused on the following aspects to determine the relationship between SPAG4, Nesprin3 and lung cancer.

Firstly, nuclear heteromorphism is an important index in determining the degree of tumor differentiation (2). The downregulation of SUN2, Nesprin1 and 2 may render the nucleus easy to deform; thus worsening the tumor cell differentiation (21). Previous studies have revealed that LINC complex proteins may regulate the proliferation and migration of cancer cells, and that Nesprin1 and 2 affect cell proliferation (15). In addition, Nesprin3 expression is required for flow-induced MTOC polarization and directional migration (19), and SUN2 inhibits tumor cell proliferation and migration (16). Although the expression of many LINC complex proteins is downregulated in cancer, SPAG4 was found to be upregulated in renal carcinoma (18). We found that the expression of SPAG4 was significantly higher in pulmonary carcinoma than in adjacent tissues. In addition, the results of immunohistochemistry revealed that SPAG4 expression was associated with tumor differentiation grade. Thus, SPAG4 may serve as a new biomarker for lung cancer diagnosis.

Secondly, the LINC complex plays a significant role in cell growth, such as cell migration, nuclear positioning, and the cell cycle (22). Knaup et al indicated that SPAG4 overexpression promoted cell proliferation (18), and our results from the scratch wound assay revealed that SPAG4 silencing inhibited the migration of cancer cells. These findings demonstrated that SPAG4 acts as a facilitator in tumor progression. Shoji et al revealed that the knockdown of SPAG4 led to tetraploidy of cancer cells, resulting in subsequent suppression of cell proliferation due to cell division disturbance (23). In the present study, we provided evidence to demonstrate that SPAG4 is a positive regulator of Nesprin3. Nesprin3, an outer nuclear membrane protein, has been reported to influence the directional migration of cells (19) and was revealed to play an important role in promoting cell migration in our study. We also examined the distribution and structure of SPAG4, and observed that SPAG4 can anchor to the inner nuclear envelope and is polarized in the cytoplasm. The SUN domain cannot be folded correctly without transmembrane helices, and may be found in the endoplasmic reticulum first, and then anchored to the inner nuclear envelope by transmembrane helices.

Thirdly, the LINC complex is known to be the link between the nuclear skeleton and cytoplasmic skeleton. The KASH and SUN family of proteins have a variety of functions in the LINC complex (24). While Nesprin1 and 2 are related to actin filaments and Nesprin 4 connects to microtubules, Nesprin 3 is 
A

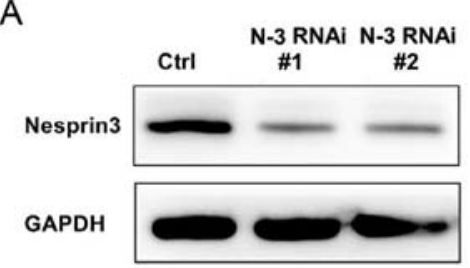

B

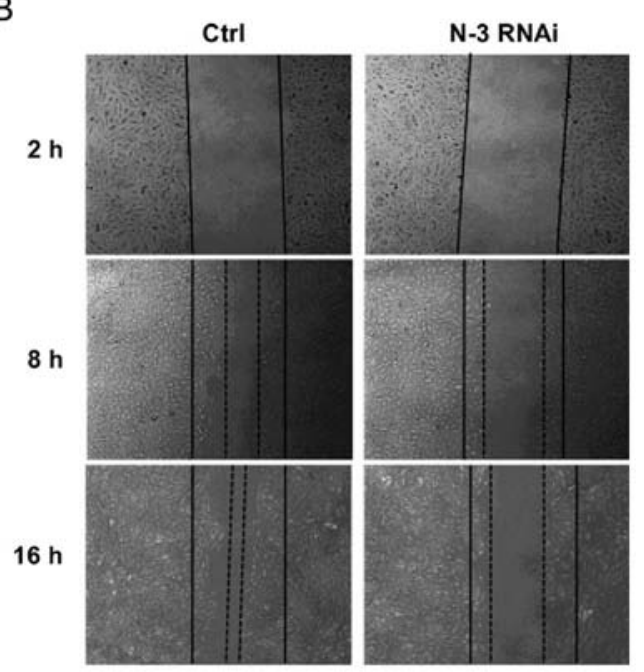

C

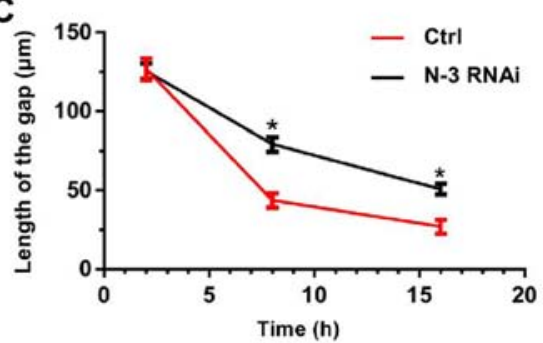

Figure 7. Effects of Nesprin 3 gene silencing. (A) Western blotting results of Nesprin 3 or GAPDH antibodies in control cells, Nesprin 3 shRNA treated cells (N-3 RNAi\#1 and N-3 RNAi\#2) at 9 days after transfection with neomycin $(1 \mathrm{mg} / \mathrm{ml})$ screening. Unrelated bands are trimmed for space. (B and C) Gap closure was significantly suppressed by silencing Nesprin3, respectively (magnification, $x 40$ ). The bars correspond to the mean \pm SEM. The P-value was calculated using the Student's t-test. "P $<0.05$. SPAG4, sperm-associated antigen 4 .

closely related to MTOC (11). The MTOC/nucleus connectivity affects a series of cell functions including migration. It has been reported that Nesprin 3 has a structural role by organizing perinuclear cytoskeletal architecture as well as a functional role by modulating flow-induced MTOC migration $(19,25)$. These results reveal the possible relationship between SPAG4 and Nesprin3. We confirmed the interaction between SPAG4 and Nesprin 3 by immunoprecipitation and BiFC assays. The silencing of SPAG4 resulted in the loose localization of Nesprin 3 and a decrease in Nesprin 3 expression. Therefore, we suggest that the roles of SPAG4 and Nesprin3 in cell migration are synergistic. Previous studies have revealed that Nesprin3 silencing reduces both the cell migration velocity and the directionality of migration (19). The scratch wound assay with Nesprin3-silenced cancer cells supports this view. Without the link between SPAG4 and Nesprin3, the MTOC/nuclear connectivity may be weakened and chromosomes may move slowly, further reducing the migration of lung cancer cells.

To maintain the core function of the LINC complex in connecting the genome and nucleoskeleton to cytoplasmic filaments and the extracellular matrix, the molecular structure of the SUN-KASH interface would need to provide a tight binding interface impervious to disruption (26). The loss of these nuclear membrane proteins leads to an incomplete nuclear membrane structure. Therefore, since a series of LINC complex proteins such as SUN2 and Nesprin2 are downregulated in tumors $(13,15,16)$, we speculate that SPAG4/Nesprin3 complexes may increase compensatorily in order to complement missing LINC complexes to maintain the total number of LINC complexes. It is possible that tumor cells have alternative and cross-coordinated mechanisms to maintain the characteristics of high invasion,once one becomes deficient, the other compensates for the lack of activity.

Finally, although this interaction was confirmed, the exact relationship between SPAG4 upregulation and the downregulation of other LINC complex proteins is still unclear. We did not determine the relationship between SPAG4 expression and the prognosis of lung cancer patients. In addition, whether the SPAG4/Nesprin3 complex complements the decreasing LINC complex in lung cancer requires further validation. Such knowledge will, in turn, provide a theoretical basis for new strategies in lung cancer diagnosis and treatment.

\section{Acknowledgements}

The authors thank Wei Li for technical assistance.

\section{Funding}

The present study was supported by the National Natural Science Foundation of China (grant nos. 81472774 and 81170615).

\section{Availability of data and materials}

The datasets used during the present study are available from the corresponding author upon reasonable request.

\section{Authors' contributions}

$\mathrm{XX}$ and $\mathrm{LJ}$ conceived the study and revised the manuscript. YJ and $\mathrm{JJ}$ designed and performed the experiments. $\mathrm{LH}$ analyzed the data and revised the manuscript. ZZ and WF assisted in the experiments. All authors read and approved the manuscript and agree to be accountable for all aspects of the research in ensuring that the accuracy or integrity of any part of the study are appropriately investigated and resolved.

\section{Ethics approval and consent to participate}

All procedures performed in the studies involving human participants were in accordance with the ethical standards of the Institutional and/or National Research Committee and the 1964 Helsinki Declaration and its later amendments or comparable ethical standards. This study was conducted with the approval of the Ethics Committee of the Third Xiangya Hospital, Central South University, Changsha, China (S071 2014). 


\section{Patient consent for publication}

Not applicable.

\section{Competing interests}

The authors declare that they have no competing interests.

\section{References}

1. Kratz JR, He J, Van Den Eeden SK, Zhu ZH, Gao W, Pham PT, Mulvihill MS, Ziaei F, Zhang $\mathrm{H}$, Su B, et al: A practical molecular assay to predict survival in resected non-squamous, non-small-cell lung cancer: Development and international validation studies. Lancet 379: 823-832, 2012.

2. Zink D, Fischer AH and Nickerson JA: Nuclear structure in cancer cells. Nat Rev Cancer 4: 677-687, 2004.

3. Nery FC, Zeng J, Niland BP, Hewett J, Farley J, Irimia D, Li Y, Wiche G, Sonnenberg A and Breakefield XO: TorsinA binds the KASH domain of nesprins and participates in linkage between nuclear envelope and cytoskeleton. J Cell Sci 121: 3476-3486, 2008

4. Ketema M and Sonnenberg A: Nesprin-3: A versatile connector between the nucleus and the cytoskeleton. Biochem Soc Trans 39: 1719-1724, 2011

5. Tapley EC and Starr DA: Connecting the nucleus to the cytoskeleton by SUN-KASH bridges across the nuclear envelope. Curr Opin Cell Biol 25: 57-62, 2013.

6. Link J, Leubner M, Schmitt J, Göb E, Benavente R, Jeang KT, $\mathrm{Xu}$ R and Alsheimer M: Analysis of meiosis in SUN1 deficient mice reveals a distinct role of SUN2 in mammalian meiotic LINC complex formation and function. PLoS Genet 10: e1004099, 2014.

7. Patel JT, Bottrill A, Prosser SL, Jayaraman S, Straatman K, Fry AM and Shackleton S: Mitotic phosphorylation of SUN1 loosens its connection with the nuclear lamina while the LINC complex remains intact. Nucleus 5: 462-473, 2014

8. Lei K, Zhang X, Ding X, Guo X, Chen M, Zhu B, Xu T, Zhuang Y, $\mathrm{Xu} \mathrm{R}$ and Han M: SUN1 and SUN2 play critical but partially redundant roles in anchoring nuclei in skeletal muscle cells in mice. Proc Natl Acad Sci USA 106: 10207-10212, 2009.

9. Thomsen C, Udhane S, Runnberg R, Wiche G, Ståhlberg A and Aman P: Fused in sarcoma (FUS) interacts with the cytolinker protein plectin: Implications for FUS subcellular localization and function. Exp Cell Res 318: 653-661, 2012.

10. Vlcek S and Foisner R: Lamins and lamin-associated proteins in aging and disease. Curr Opin Cell Biol 19: 298-304, 2007.

11. Fridkin A, Penkner A, Jantsch V and Gruenbaum Y: SUN-domain and $\mathrm{KASH}$-domain proteins during development, meiosis and disease. Cell Mol Life Sci 66: 1518-1533, 2009.

12. Kennedy C, Sebire K, de Kretser DM and O'Bryan MK: Human sperm associated antigen 4 (SPAG4) is a potential cancer marker. Cell Tissue Res 315: 279-283, 2004.

13. Marmé A,Zimmermann HP, Moldenhauer G,Schorpp-KistnerM, Müller C, Keberlein O, Giersch A, Kretschmer J, Seib B, Spiess E, et al: Loss of Drop1 expression already at early tumor stages in a wide range of human carcinomas. Int J Cancer 123: 2048-2056, 2008.
14. Tessema M, Willink R, Do K, Yu YY, Yu W, Machida EO, Brock M, Van Neste L, Stidley CA, Baylin SB, et al: Promoter methylation of genes in and around the candidate lung cancer susceptibility locus 6q23-25. Cancer Res 68: 1707-1714, 2008.

15. Matsumoto A, Hieda M, Yokoyama Y, Nishioka Y, K, Tsujimoto $\mathrm{M}$ and Matsuura $\mathrm{N}$ : Global loss of a nuclear lamina component, lamin A/C, and LINC complex components SUN1, SUN2, and nesprin-2 in breast cancer. Cancer Med 4: 1547-1557, 2015.

16. Lv XB, Liu L, Cheng C, Yu B, Xiong L, Hu K, Tang J, Zeng L and Sang Y: SUN2 exerts tumor suppressor functions by suppressing the Warburg effect in lung cancer. Sci Rep 5: 17940, 2015.

17. Tarnasky H, Gill D, Murthy S, Shao X, Demetrick DJ and van der Hoorn FA: A novel testis-specific gene, SPAG4, whose product interacts specifically with outer dense fiber protein ODF27, maps to human chromosome 20q11.2. Cytogenet Cell Genet 81: 65-67, 1998.

18. Knaup KX, Monti J, Hackenbeck T, Jobst-Schwan T, Klanke B, Schietke RE, Wacker I, Behrens J, Amann K, Eckardt KU, et al: Hypoxia regulates the sperm associated antigen 4 (SPAG4) via HIF, which is expressed in renal clear cell carcinoma and promotes migration and invasion in vitro. Mol Carcinog 53: 970-978, 2014

19. Morgan JT, Pfeiffer ER, Thirkill TL, Kumar P, Peng G, Fridolfsson HN, Douglas GC, Starr DA and Barakat AI: Nesprin-3 regulates endothelial cell morphology, perinuclear cytoskeletal architecture, and flow-induced polarization. Mol Biol Cell 22: 4324-4334, 2011.

20. Yeh CH, Kuo PL, Wang YY, Wu YY, Chen MF, Lin DY, Lai TH, Chiang HS and Lin YH: SEPT12/SPAG4/LAMINB1 complexes are required for maintaining the integrity of the nuclear envelope in postmeiotic male germ cells. PLoS One 10: e0120722, 2015.

21. Cartwright $S$ and Karakesisoglou I: Nesprins in health and disease. Semin Cell Dev Biol 29: 169-179, 2014.

22. Isermann $P$ and Lammerding J: Nuclear mechanics and mechanotransduction in health and disease. Curr Biol 23: R1113-R1121, 2013.

23. Shoji K, MurayamaT, Mimura I, Wada T, Kume H, Goto A, Ohse T, Tanaka T, Inagi R, van der Hoorn FA, et al: Sperm-associated antigen 4 , a novel hypoxia-inducible factor 1 target, regulates cytokinesis, and its expression correlates with the prognosis of renal cell carcinoma. Am J Pathol 182: 2191-2203, 2013.

24. Jungwirth MT, Kumar D, Jeong DY and Goodchild RE: The nuclear envelope localization of DYT1 dystonia torsin $\mathrm{A}-\Delta \mathrm{E}$ requires the SUN1 LINC complex component. BMC Cell Biol 12: 24, 2011

25. Revach OY, Weiner A, Rechav K, Sabanay I, Livne A and Geiger B: Mechanical interplay between invadopodia and the nucleus in cultured cancer cells. Sci Rep 5: 9466, 2015.

26. Meinke $P$ and Schirmer EC: LINC'ing form and function at the nuclear envelope. FEBS Lett 589 (19 Pt A): 2514-2521, 2015.

This work is licensed under a Creative Commons Attribution-NonCommercial-NoDerivatives 4.0 International (CC BY-NC-ND 4.0) License. 